\title{
Upper critical field in the model with finite-range interaction between electrons
}

\author{
A. V. Shumilin, ${ }^{1}$ V. V. Baranov, ${ }^{2}$ and V. V. Kabanov ${ }^{3}$ \\ ${ }^{1}$ Ioffe Institute, 194021 St.-Petersburg, Russia \\ ${ }^{2}$ Department of Physics, University of Antwerp, Groenenborgerlaan 171, 2020 Antwerp, Belgium \\ ${ }^{3}$ Department for Complex Matter, Jozef Stefan Institute, 1001 Ljubljana, Slovenia
}

(Received 2 August 2016; revised manuscript received 10 October 2016; published 14 November 2016)

\begin{abstract}
We develop a theory of the upper critical field in a BCS superconductor with a nonlocal interaction between electrons. We have shown that the nonlocal interaction is characterized by the parameter $k_{F} \rho_{0}$, where $k_{F}$ is the Fermi momentum and $\rho_{0}$ is the radius of electron-electron interaction. The presence of the external magnetic field leads to the generation of additional components of the order parameter with different angular momenta. This effect leads to the enhancement of the upper critical field above the orbital limiting field. In addition the upward curvature in the temperature dependence of $H_{c 2}(T)$ in the clean limit is predicted. The impurity scattering suppresses the effect in the dirty limit.
\end{abstract}

DOI: 10.1103/PhysRevB.94.174506

\section{INTRODUCTION}

The upper critical field $H_{c 2}$ is one of the important characteristics of type-II superconductors. When the field is sufficiently high, the superconductivity is destroyed, and the field uniformly penetrates to the sample. With the continuous decrease of the field superconducting regions start to nucleate spontaneously at the upper critical filed $H=H_{c 2}(T)$. Since within these regions the order parameter is small, the linearized phenomenological Ginzburg-Landau equations are applicable in the vicinity of zero-field $T_{c}$. This leads to the linear in $T$ upper critical field $H_{c 2}(T) \propto T_{c}-T$ [1]. At zero temperature, $H_{c 2}(0)$ is normally below the Clogston-Chandrasekhar [2] or the Pauli pair-breaking limit given by $H_{p}=1.84 T_{c}$ (in teslas) for the singlet pairing.

Recent high-magnetic-field studies in cuprates [3-6], $\mathrm{MgB}_{2}, \mathrm{Ba}_{1-x} \mathrm{~K}_{x} \mathrm{BiO}_{3}$, and pnictide superconductors [7-9], spin ladders [10], and organic superconductors [11] have revealed a non-BCS upward curvature of the resistive $H_{c 2}(T)$. In some cases [11-13] the Pauli limit was exceeded by several times. A nonlinear temperature dependence in the vicinity of $T_{c}$ has been unambiguously observed in a few samples $[6-8,12,14,15]$. The observation of the departure from the BCS behavior creates some controversy in the interpretation of the resistive critical field [16-18]. If in some cases there is little doubt that the resistive transition corresponds to the upper critical field $H_{c 2}[6-8,10-12,14,15]$, some measurements on high-temperature superconductors suggest that the real upper critical field is much higher than the resistive transition [16-18]. Indeed, the thermodynamic determination of $H_{c 2}$ [18-20] and anomalous diamagnetism above the resistive transition [16,21] seem to justify such a conclusion.

Several theoretical concepts have been proposed in order to explain a non-BCS upward curvature of the resistive $H_{c 2}(T)$. Some of the concepts are based on the fact that the size of the pairs is smaller than the average distance between electrons, and therefore the superconducting state may be approximated by the weakly interacting charged Bose gas [14,22]. In that case the upper critical field has universal temperature dependence $\left(T_{c}-T\right)^{3 / 2}$ near $T_{c}[14,22]$.

Another approach is based on the multiband picture $[7,15]$. In the case when the Fermi surface has few sheets, the gaps on different branches of the Fermi surface are independent functions with different coupling constants. In such a system the upper critical field may deviate considerably from the classical BCS behavior, leading to the weak upward curvature in the temperature dependence of $H_{c 2}[7,15]$.

Similar temperature dependence of the upper critical field may be caused by the field-induced mixture of the superconducting gaps of different symmetries [23-25]. On the basis of the phenomenological Ginzburg-Landau approach it has been suggested that in addition to the $d$-wave gap the external field may lead to generation of the $s$-wave [23], additional $d$-wave [24], or $p$-wave [25] components of the order parameter. This effect is caused by the fact that symmetry allows nontrivial gradient terms in the free energy $[23,24]$, including Lifshitz invariants [25]. This type of coupling may also lead to unusual temperature dependence of the upper critical field $H_{c 2}(T)$ $[23,25]$. This type of Ginzburg-Landau equation was later derived from Gorkov equations assuming nonlocal potential between electrons [26]. Note that the approach based on the Ginzburg-Landau equation is restricted to the relatively high temperatures $T_{c}-T \ll T_{c}$.

Here we generalize Werthamer-Helfand-Hohenberg (WHH) theory [27-29] to the case of nonlocal interaction between electrons. It allows us to go beyond the high-temperature limit and consider the upper critical field behavior caused by the intermixture of the different order parameters at arbitrary temperature. We demonstrate that the nonlocal interaction is characterized by the parameter $k_{F} \rho_{0}$, where $k_{F}$ is the Fermi momentum and $\rho_{0}$ is the radius of electron-electron interaction. We show that in the presence of the external magnetic field the finite radius of electron-electron interaction leads to the generation of additional components of the order parameter with different angular dependence. This effect leads to the enhancement of the upper critical field above the orbital limiting field as well as to the upward curvature in the temperature dependence of $H_{c 2}(T)$ in the clean limit. The increase in the impurity concentration suppresses the effect.

\section{MAIN EQUATIONS}

We consider a superconductor with a nonlocal pairing potential $V\left(\mathbf{r}-\mathbf{r}^{\prime}\right)$ that explicitly depends on coordinates $\mathbf{r}, \mathbf{r}^{\prime}$ 
and can be characterized by the radius of interaction $\rho_{0}$. The superconductor can be described by the Hamiltonian

$$
\begin{aligned}
H= & \sum_{\sigma=\uparrow, \downarrow} \int d \mathbf{r} \Psi_{\sigma}^{\dagger}(\mathbf{r}) \hat{\xi} \Psi_{\sigma}(\mathbf{r}) \\
& +\int d \mathbf{r} d \mathbf{r}^{\prime} V\left(\mathbf{r}-\mathbf{r}^{\prime}\right) \Psi_{\uparrow}^{\dagger}(\mathbf{r}) \Psi_{\downarrow}^{\dagger}\left(\mathbf{r}^{\prime}\right) \Psi_{\downarrow}\left(\mathbf{r}^{\prime}\right) \Psi_{\uparrow}(\mathbf{r}) .
\end{aligned}
$$

Here $\hat{\xi}=\frac{1}{2 m^{*}}(i \nabla+e \mathbf{A})^{2}-\mu, m^{*}$ is the effective mass of an electron, $\mu$ is the chemical potential, and $\mathbf{A}$ is the vector potential.

The equation of motion for the field operator $\Psi_{\uparrow}(\mathbf{r}, t)$ derived from the Hamiltonian (1) is

$$
\left(\frac{\partial}{\partial t}+\hat{\xi}\right) \Psi_{\uparrow}(\mathbf{r}, t)=-\int d \mathbf{r}^{\prime \prime} \Delta\left(\mathbf{r}, \mathbf{r}^{\prime \prime}\right) \bar{\Psi}_{\downarrow}\left(\mathbf{r}^{\prime \prime}, t\right),
$$

where $\Delta\left(\mathbf{r}, \mathbf{r}^{\prime}\right)=V\left(\mathbf{r}-\mathbf{r}^{\prime}\right)\left\langle\Psi_{\downarrow}\left(\mathbf{r}^{\prime}\right) \Psi_{\uparrow}(\mathbf{r})\right\rangle$ is the order parameter of the superconductor. In contrast to the BCS model the order parameter explicitly depends on two coordinates, $\mathbf{r}$ and $\mathbf{r}^{\prime}$.

Multiplying Eq. (2) by $\Psi_{\downarrow}\left(\mathbf{r}^{\prime}, t^{\prime}\right)$, we obtain the equation for the anomalous Green's function $F\left(\mathbf{r} t, \mathbf{r}^{\prime} t^{\prime}\right)$. We write this equation in terms of Matsubara frequencies $\omega_{n}=\pi T(2 n+1)$, where $n$ is an integer,

$$
\left(-i \omega_{n}+\hat{\xi}\right) F_{\omega_{n}}\left(\mathbf{r}, \mathbf{r}^{\prime}\right)=\int d \mathbf{r}^{\prime \prime} \Delta\left(\mathbf{r}, \mathbf{r}^{\prime \prime}\right) G_{-\omega_{n}}\left(\mathbf{r}^{\prime}, \mathbf{r}^{\prime \prime}\right) .
$$

The anomalous Green's function is closely related to the order parameter

$$
\Delta\left(\mathbf{r}, \mathbf{r}^{\prime}\right)=V\left(\mathbf{r}-\mathbf{r}^{\prime}\right) T \sum_{\omega_{n}} F_{\omega_{n}}\left(\mathbf{r}, \mathbf{r}^{\prime}\right) .
$$

In the present study we are interested in the upper critical field. The order parameter at the magnetic field close to critical is small. It allows us to use a normal-state expression for the normal Green's function $G_{\omega_{n}}\left(\mathbf{r}, \mathbf{r}^{\prime}\right)$ [30].

Expressions (3) and (4) allow us to give a closed equation for the order parameter in terms of the normal-state Green's functions,

$$
\begin{aligned}
\Delta(\boldsymbol{\rho}, \mathbf{R})= & -T V(\boldsymbol{\rho}) \sum_{n} \int G_{\omega_{n}}\left(\mathbf{R}+\boldsymbol{\rho} / 2, \mathbf{R}^{\prime}+\boldsymbol{\rho}^{\prime} / 2\right) \\
& \times \Delta\left(\boldsymbol{\rho}^{\prime}, \mathbf{R}^{\prime}\right) G_{-\omega_{n}}\left(\mathbf{R}-\boldsymbol{\rho} / 2, \mathbf{R}^{\prime}-\boldsymbol{\rho}^{\prime} / 2\right) d \boldsymbol{\rho}^{\prime} d \mathbf{R}^{\prime} .
\end{aligned}
$$

Here we introduce a new set of variables $\mathbf{R}$ and $\rho$ for the order parameter $\Delta$ and the pairing potential $V$. This variables are related to the variables $\mathbf{r}$ and $\mathbf{r}^{\prime}$ used in Eq. (4) as follows. The variable $\mathbf{R}=\left(\mathbf{r}+\mathbf{r}^{\prime}\right) / 2$ describes the motion of the center of mass of the Cooper pair. It is related to the macroscopic distribution of the order parameter in the sample. At zero magnetic field the order parameter is uniform and does not depend on $\mathbf{R}$. The variable $\rho=\mathbf{r}-\mathbf{r}^{\prime}$ describes the relative motion of electrons in the Cooper pair. It appears due to the nonlocal pairing potential and describes the symmetry of the order parameter. If we select local pairing potential $V \propto \delta(\rho), \rho$ should always be equal to zero. Our theory is reduced to the conventional BCS theory in this case. In the more general case the important values of $\rho$ are of the order of $\rho_{0}$.
The internal coordinate $\rho$ allows an additional degree of freedom to the superconductivity. We are especially interested in the angular dependence of $\rho$. We will show that the order parameter can be divided into the components related to the different angular momenta of $\boldsymbol{\rho}$. These components are independent without the external magnetic field. In the magnetic field these components are intermixed. This intermixing leads to the upward curvature of $H_{c 2}(T)$ dependence. In the present study we focus on the two-dimensional (2D) case. It may be realized experimentally in atomically thin films or in very anisotropic superconductors similar to high- $T_{c}$ superconductors. In this case the components of the order parameter can be classified by the projection of the angular momentum on the axis perpendicular to the $2 \mathrm{D}$ plane. In principle a similar theory can be formulated in three dimensions when the components of the order parameter are classified by the value of the angular momentum. However, this issue goes beyond of the present study.

\section{CLEAN LIMIT}

We start our consideration from the clean limit $(l \gg \xi)$, where the mean free path $l$ is much larger than the coherence length $\xi$ of the superconductor. In this case we neglect the effects of the impurities, and we can give an explicit expression for the Green's function:

$$
G_{\omega_{n}}^{(0)}(\mathbf{R})= \begin{cases}\frac{-i m^{*}}{\sqrt{2 \pi k_{F} R}} e^{i\left(k_{F} R-\pi / 4\right)-\frac{\left|\omega_{n}\right|}{v_{F}} R}, & \omega_{n}>0, \\ \frac{i m^{*}}{\sqrt{2 \pi k_{F} R}} e^{-i\left(k_{F} R-\pi / 4\right)-\frac{\left|\omega_{n}\right|}{v_{F}} R}, & \omega_{n}<0 .\end{cases}
$$

Here we consider the limit $k_{F} R \gg 1$. Expression (6) corresponds to the normal Green's function without magnetic field. The Green's function in the magnetic field is related to $G_{\omega_{n}}^{(0)}$ as $G_{\omega_{n}}\left(\mathbf{R}, \mathbf{R}^{\prime}\right)=G_{\omega_{n}}^{(0)}\left(\mathbf{R}^{\prime}-\mathbf{R}\right) \exp \left[i \phi\left(\mathbf{R}, \mathbf{R}^{\prime}\right)\right]$, where $\phi\left(\mathbf{R}, \mathbf{R}^{\prime}\right) \approx e \int_{\mathbf{R}}^{\mathbf{R}^{\prime}} d \mathbf{s} \mathbf{A}(\mathbf{s})$. We also neglect the paramagnetic effects, assuming that they are as small as $\Delta / E_{F} \ll 1$. These effects may be important in the case where the upper critical field reaches the Pauli pair-breaking limit. This situation usually occurs due to strong impurity scattering. However, as will be shown later, strong impurity scattering suppresses the field-induced mixture of pairing with different angular momenta.

The equation for the order parameter with the Green's functions (6) is

$$
\begin{aligned}
\Delta(\boldsymbol{\rho}, \mathbf{R})= & -V(\boldsymbol{\rho}) \int d \mathbf{R}^{\prime} d \boldsymbol{\rho}^{\prime} \mathcal{K}_{0}(\widetilde{R}) \Delta\left(\boldsymbol{\rho}^{\prime}, \mathbf{R}^{\prime}\right) \\
& \times \exp \left[2 i \phi\left(\mathbf{R}, \mathbf{R}^{\prime}\right)\right] \cos \left(\frac{k_{F} \widetilde{\mathbf{R}} \varrho}{\widetilde{R}}\right),
\end{aligned}
$$

where $\widetilde{\mathbf{R}}=\mathbf{R}^{\prime}-\mathbf{R}, \widetilde{R}=|\widetilde{\mathbf{R}}|, \varrho=\rho^{\prime}-\rho$, and the kernel

$$
\mathcal{K}_{0}(R)=\frac{m^{* 2} T}{2 \pi k_{F} R \sinh \left(2 \pi T R / v_{F}\right)} .
$$

Let us assume for simplicity that the pairing potential has the form $V(\rho)=-V_{0} \delta\left(|\rho|-\rho_{0}\right)$, where $\rho_{0}$ plays the role of the interaction radius. The potential with the finite range of interaction leads to the formation of pairs with different angular momenta. Indeed, Fourier components of the potential $V\left(\mathbf{k}^{\prime}-\right.$ $\mathbf{k})=V\left(\varphi^{\prime}, \varphi\right)$, where $k, k^{\prime}=k_{F}$, depend only on polar angles 
$\varphi, \varphi^{\prime}$ of vectors $\mathbf{k}, \mathbf{k}^{\prime}$. Calculating the matrix elements $V_{n, n^{\prime}}=$ $\int d \varphi d \varphi^{\prime} V\left(\varphi^{\prime}, \varphi\right) e^{i n \varphi} e^{-i n^{\prime} \varphi^{\prime}}$, we obtain

$$
V_{n, n^{\prime}}=-V_{0} \rho_{0} J_{n}^{2}\left(k_{F} \rho_{0}\right) \delta_{n, n^{\prime}} .
$$

Here $J_{n}(x)$ is the Bessel function. Therefore this potential leads to the pairing in the channels with nonzero orbital moments $n$ with the critical temperatures $T_{c n}=T_{c 0} \exp \left(\lambda_{0}^{-1}-\lambda_{n}^{-1}\right)$ and $\lambda_{n}=\frac{m^{*}}{2 \pi} V_{0} \rho_{0} J_{n}^{2}\left(k_{F} \rho_{0}\right)$. Note that the strength of the pairing is determined by the parameter $k_{F} \rho_{0}$. If $k_{F} \rho_{0} \ll 1$, all channels except the $n=0$ channel are suppressed because $J_{n}(x) \approx x^{n}$ when $x \ll 1$. When $k_{F} \rho_{0} \gtrsim 1$, one of the channels with $n \neq 0$ can have the largest $T_{c}$ and corresponds to the main order parameter of the superconductor [31]. In real systems the smallest radius of interaction is determined by the screening radius, and therefore the situation $k_{F} \rho_{0}>1$ seems to be natural. Like in the conventional BCS theory we do not try to choose a realistic pairing potential. Real interaction between electrons is complicated and may not be characterized by the potential. On the other hand, we believe that the exact form of the potential is not very important for our results. All the final results will be expressed in terms of critical temperatures $T_{c n}$ in different pairing channels [see Eqs. (19), (21), and (29)]. Therefore the exact form of the potential drops out from our results. Also we want to note that as long as the pairing potential acts only on the electrons near the Fermi surface it can be reduced to its dependence on angles $\varphi, \varphi^{\prime}$. This dependence defines $V_{n, n^{\prime}}$ in the Eq. (9) and the critical temperatures $T_{c n}$. We believe that any coordinate form of the potential that results in the same temperatures $T_{c n}$ should lead to similar results for $H_{c 2}$. Here we choose the potential to make the calculations as simple as possible.

For the discussed choice of the pairing potential the order parameter can be written as

$$
\Delta(\boldsymbol{\rho}, \mathbf{R})=\Delta\left(\rho_{0}, \varphi, \mathbf{R}\right)=\sum_{n} \Delta_{n}(\mathbf{R}) e^{i n \varphi} .
$$

Substituting this expansion back into the integral equation (7), we obtain

$$
\begin{aligned}
\Delta_{n}(\mathbf{R})= & V_{0} \rho_{0} \sum_{n^{\prime}} \int d \mathbf{R}^{\prime} \mathcal{K}_{0}(\widetilde{R}) L_{n n^{\prime}}(\widetilde{\mathbf{R}}) \\
& \times \exp \left[2 i \phi\left(\mathbf{R}, \mathbf{R}^{\prime}\right)\right] \Delta_{n^{\prime}}\left(\mathbf{R}^{\prime}\right),
\end{aligned}
$$

where the matrix $L_{n n^{\prime}}$ is defined as

$$
L_{n, n^{\prime}}=e^{i\left(n^{\prime}-n\right) \theta}\left\{\begin{array}{l}
(-1)^{l+k} J_{2 l}\left(k_{F} \rho_{0}\right) J_{2 k}\left(k_{F} \rho_{0}\right), \\
|n|=2 l,\left|n^{\prime}\right|=2 k, \\
(-1)^{l+k} J_{2 l+1}\left(k_{F} \rho_{0}\right) J_{2 k+1}\left(k_{F} \rho_{0}\right), \\
|n|=2 l+1,\left|n^{\prime}\right|=2 k+1,
\end{array}\right.
$$

where $\theta$ is the polar angle of the vector $\widetilde{\mathbf{R}}$.

The matrix $L_{n, n^{\prime}}$ connects the angular momenta of the internal coordinate $\rho$ and the averaged coordinate $\mathbf{R}$. While the total momentum is conserved, the nondiagonal matrix elements of $L_{n, n^{\prime}}$ allow the transfer of the momentum between the degrees of freedom corresponding to $\rho$ and $\mathbf{R}$.

Following the procedure described in Ref. [28], we expand $\Delta\left(\mathbf{R}^{\prime}\right)$ into the series over $\mathbf{R}^{\prime}-\mathbf{R}$ and join the space derivatives with vector potential into the single operator $\mathbf{D}=\frac{\partial}{\partial \mathbf{R}}+2 i e \mathbf{A}$ :

$$
\Delta_{n}(\mathbf{R})=V_{0} \rho_{0} \sum_{n^{\prime}} \int d \widetilde{\mathbf{R}} \mathcal{K}_{0}(\widetilde{R}) L_{n n^{\prime}}(\widetilde{\mathbf{R}}) \exp (\mathbf{D} \cdot \widetilde{\mathbf{R}}) \Delta_{n^{\prime}}(\mathbf{R}) .
$$

Note that the operator $\mathbf{D}$ acts only on the coordinate $\mathbf{R}$.

Let us choose the gauge $\mathbf{A}=(0, H x, 0)$. With this gauge the order parameters $\Delta_{n}$ are not dependent on $y$ and can be considered as functions $\Delta_{n}(x)$. Moreover, this gauge allows us to relate different terms $\Delta_{n}$ of the order parameter to different coordinate functions $\psi_{m}$,

$$
\psi_{m}=\left(\frac{1}{\pi \hbar L_{H}^{2}}\right)^{1 / 4} \frac{1}{\sqrt{2^{m} m !}} e^{-x^{2} / 2 L_{H}^{2}} H_{m}\left(\frac{x}{L_{H}}\right),
$$

that correspond to the eigenfunctions of the harmonic oscillator. Here $L_{H}^{-1}=\sqrt{\frac{2 \pi H}{\phi_{0}}}$ is the magnetic length, and $\phi_{0}$ is the flux quantum. $H_{n}$ are the Hermite polynomials.

To apply the basis (14) it is useful to make the expansion $\mathbf{D} \widetilde{\mathbf{R}}=\frac{\widetilde{R}}{2}\left(D_{+} e^{-i \theta}+D_{-} e^{i \theta}\right)$, where $D_{ \pm}=D_{x} \pm i D_{y}$ and $\theta$ is the polar angle of the vector $\widetilde{\mathbf{R}}$. The action of the operators $D_{-}$ and $D_{+}$on the functions $\Psi_{m}$ has a simple form,

$$
\begin{aligned}
& D_{-} \psi_{m}(x)=\frac{\sqrt{2 m}}{L_{H}} \psi_{m-1}(x), \\
& D_{+} \psi_{m}(x)=-\frac{\sqrt{2(m+1)}}{L_{H}} \psi_{m+1}(x) .
\end{aligned}
$$

Equation (13) in the notation $D_{+}, D_{-}$has the form

$$
\begin{aligned}
\Delta_{n}(\mathbf{R})= & V_{0} \rho_{0} \sum_{n^{\prime}} \int d \widetilde{\mathbf{R}} \mathcal{K}_{0}(\widetilde{R}) L_{n n^{\prime}}(\widetilde{\mathbf{R}}) \exp \left(-\frac{\widetilde{R}^{2}}{4 L_{H}^{2}}\right) \\
& \times \exp \left(\frac{\widetilde{R}}{2} e^{-i \theta} D_{+}\right) \exp \left(\frac{\widetilde{R}}{2} e^{i \theta} D_{-}\right) \Delta_{n^{\prime}}(\mathbf{R}) .
\end{aligned}
$$

Here we have used the formula $e^{(\hat{P}+\hat{Q})}=e^{\hat{P}} e^{\hat{Q}} e^{-[\hat{P}, \hat{Q}] / 2}$.

Expressions (15) allow us to search for the solution of this equation in the form

$$
\Delta_{n}(\mathbf{R})=\Delta_{n} \psi_{n+m_{0}}(x)
$$

The constant $m_{0}$ corresponds to the dominant pairing channel that has the largest critical temperature $T_{c}$.

The coordinate dependence (17) reduces the integral equation (16) to a matrix equation. The size of the matrix is formally infinite. However, in a realistic situation one can easily apply a cutoff for the size of the matrix. The order parameter $\Delta_{n}$ is always related to the Bessel function $J_{n}\left(k_{F} \rho_{0}\right)$. For realistic $\rho_{0}$ these Bessel functions are small for large $n$, which allows us to neglect $\Delta_{n}$ with large $n$.

\section{A. The $n=0$ dominant channel}

Let us first consider the case when the $n=0$ channel is dominant. This means that $T_{c 0}>T_{c n}$ for $n \neq 0$. On the other hand, $T_{c n}$ for $n \neq 0$ should be large enough to have a relatively large effect on $H_{c 2}(T)$. This situation takes place when $k_{F} \rho_{0} \approx$ 2 . In this case it is sufficient to study the admixture of the order parameter $\Delta_{2}$ in the channel $n=2$.

The dominant $n=0$ channel corresponds to $m_{0}=0$. The order parameter $\Delta_{0}$ has the coordinate dependence $\psi_{0}$, and the order parameter $\Delta_{2}$ has the coordinate dependence $\psi_{2}$. This 


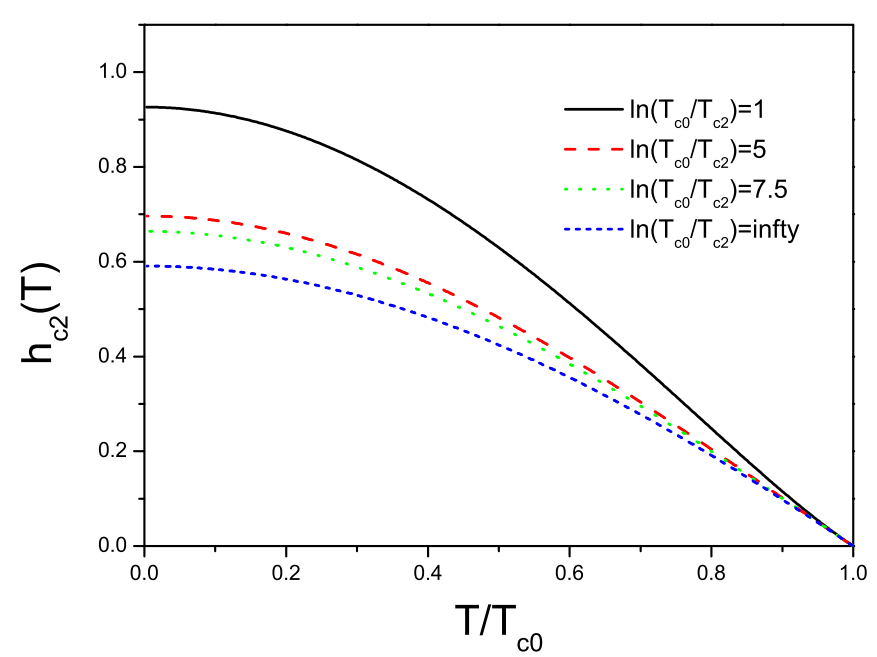

FIG. 1. Upper critical field $h_{c 2}=\frac{H_{c 2}(T)}{T_{c 0} d H_{c 2}\left(T_{c 0}\right) / d T}$ for different ratios of $T_{c 0} / T_{c 2}$ in the case when the pairing in the channel $n=0$ is dominant.

dependence leads to the matrix equation

$$
\left(\begin{array}{lc}
I_{1} L_{00} & -I_{3} L_{02} \\
-I_{3} L_{20} & I_{2} L_{22}
\end{array}\right)\left(\begin{array}{c}
\Delta_{0} \\
\Delta_{2}
\end{array}\right)=0
$$

where

$$
\begin{aligned}
& I_{1}=\ln \frac{T}{T_{c 0}}+\int_{0}^{\infty} d x \frac{1-\exp \left(-\frac{x^{2}}{4 z}\right)}{\sinh (x)}, \\
& I_{2}=\ln \frac{T}{T_{c 2}}+\int_{0}^{\infty} d x \frac{1-\exp \left(-\frac{x^{2}}{4 z}\right)\left(1-\frac{x^{2}}{z}+\frac{x^{4}}{8 z^{2}}\right)}{\sinh (x)}, \\
& I_{3}=\int_{0}^{\infty} d x \frac{x^{2}}{2 \sqrt{2} z} \frac{\exp \left(-\frac{x^{2}}{4 z}\right)}{\sinh (x)},
\end{aligned}
$$

$z=\frac{2 \pi T^{2} \phi_{0}}{v_{F}^{2} H}, L_{00}=J_{0}^{2}\left(k_{F} \rho_{0}\right), L_{22}=J_{2}^{2}\left(k_{F} \rho_{0}\right), \quad$ and $\quad L_{02}=$ $L_{20}=J_{0}\left(k_{F} \rho_{0}\right) J_{2}\left(k_{F} \rho_{0}\right)$. Equation (18) has a solution if $I_{1} I_{2}-I_{3}^{2}=0$. Therefore all the details about the pairing potential are dropped out of the equation for $H_{c 2}$. The only information about the potential remains in the critical temperature in the channel with $n=0, T_{c 0}$ and in the channel with $n=2, T_{c 2}$.

To calculate the critical field from Eq. (18) one should find the maximal magnetic field $H$ when the equation has a nontrivial solution at the given temperature. The calculated critical fields for this case are presented in Fig. 1. The main effect due to admixture of the component of the gap with $n=2$ is the increase of the critical field up to $50 \%$. Moreover, the upward curvature of the temperature dependence $H_{c 2}(T)$ is also clearly pronounced.

\section{B. The $n=2$ dominant channel}

When the parameter $k_{F} \rho_{0}$ increases $k_{F} \rho_{0} \approx 3.5, T_{c 2}>T_{c 0}$, and the dominant channel is the one with $n=2$. In this situation one should choose $m_{0}=2$, and the magnetic field leads to the coupling between the channels with $n=-2,0,2$.

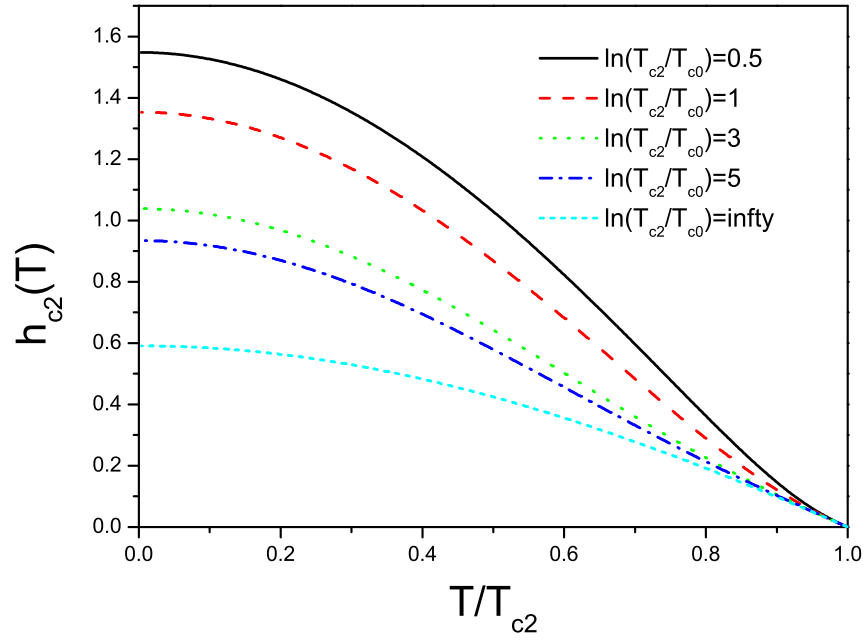

FIG. 2. Upper critical field $h_{c 2}=\frac{H_{c 2}(T)}{T_{c 2} d H_{c 2}\left(T_{c 2}\right) / d T}$ for different ratios of $T_{c 2} / T_{c 0}$ in the case when the pairing in the channel $n=2$ is dominant.

The system of equations for the order parameter reads

$$
\left(\begin{array}{lcc}
I_{11} L_{22} & -I_{12} L_{20} & -I_{13} L_{22} \\
-I_{21} L_{02} & I_{22} L_{00} & -I_{23} L_{02} \\
-I_{31} L_{22} & -I_{32} L_{20} & I_{33} L_{22}
\end{array}\right)\left(\begin{array}{l}
\Delta_{-2} \\
\Delta_{0} \\
\Delta_{2}
\end{array}\right)=0,
$$

where

$$
\begin{aligned}
& I_{11}=\ln \frac{T}{T_{c 2}}+\int_{0}^{\infty} d x \frac{1-\exp \left(-\frac{x^{2}}{4 z}\right)}{\sinh (x)}, \\
& I_{22}=\ln \frac{T}{T_{c 0}}+\int_{0}^{\infty} d x \frac{1-\exp \left(-\frac{x^{2}}{4 z}\right)\left(1-\frac{x^{2}}{z}+\frac{x^{4}}{8 z^{2}}\right)}{\sinh (x)}, \\
& I_{33}=\ln \frac{T}{T_{c 2}}+\int_{0}^{\infty} d x \frac{1-\exp \left(-\frac{x^{2}}{4 z}\right)\left(1-\frac{2 x^{2}}{z}+\frac{3 x^{4}}{4 z^{2}}-\frac{x^{6}}{12 z^{3}}+\frac{x^{8}}{384 z^{4}}\right)}{\sinh (x)}, \\
& I_{12}=I_{21}=\int_{0}^{\infty} d x \frac{x^{2}}{2 \sqrt{2} z} \frac{\exp \left(-\frac{x^{2}}{4 z}\right)}{\sinh (x)}, \\
& I_{13}=I_{31}=\int_{0}^{\infty} d x \frac{\sqrt{6} x^{4}}{48 z^{2}} \frac{\exp \left(-\frac{x^{2}}{4 z}\right)}{\sinh (x)} \\
& I_{23}=I_{32}=\frac{\sqrt{3}}{2} \int_{0}^{\infty} d x\left(\frac{x^{2}}{z}-\frac{x^{4}}{3 z^{2}}+\frac{x^{6}}{48 z^{3}}\right) \frac{\exp \left(-\frac{x^{2}}{4 z}\right)}{\sinh (x)} .
\end{aligned}
$$

Again the upper critical field is determined from the equation $\operatorname{Det}(\hat{I})=0$, where matrix $\hat{I}$ is the left-hand-side matrix in Eq. (20). All the details of the potential are hidden in critical temperatures $T_{c 0}$ and $T_{c 2}$.

Figure 2 represents the upper critical field calculated for this case. As can be seen from this picture, there is a strong enhancement of the critical field $H_{c 2}(0)$. Usually, $H_{c 2}(0)$ is expressed via the slope of the critical field at $T_{c}$ (the orbital limiting field) [32]:

$$
H_{c 2}(0)=\left.0.69 T_{c} \frac{d H_{c 2}(T)}{d T}\right|_{T=T_{c}} .
$$




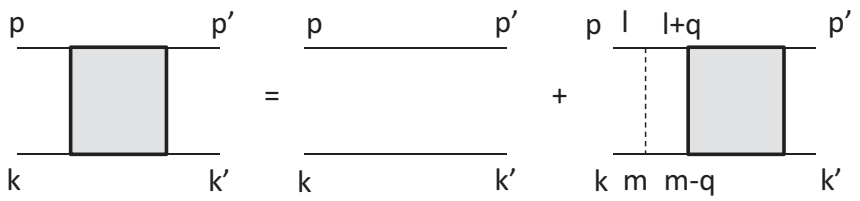

FIG. 3. Diagrammatic representation for the kernel $K\left(\mathbf{p}, \mathbf{p}^{\prime}, \mathbf{k}, \mathbf{k}^{\prime}\right)$, where the dashed line represents impurity scattering.

In the considered case $H_{c 2}(0)$ is strongly enhanced in comparison with the orbital limiting field (22). The upward curvature of $H_{c 2}(T)$ is even more pronounced than in Fig. 1.

\section{SUPERCONDUCTOR WITH IMPURITIES}

In order to describe superconducting pairing in the presence of impurities we need to average the product of two Green's functions in Eq. (5) over impurities. This averaging may be described by the diagrammatic equation (Fig. 3). As presented in Fig. 3, the corresponding integral equation contains singleparticle Green's functions averaged over impurities as well as the renormalized vertex $[30,33]$,

$$
\begin{aligned}
& K_{\omega}\left(\mathbf{p}, \mathbf{p}^{\prime}, \mathbf{k}, \mathbf{k}^{\prime}\right)=K_{\omega}^{0}\left(\mathbf{p}, \mathbf{p}^{\prime}, \mathbf{k}, \mathbf{k}^{\prime}\right)+\frac{n}{(2 \pi)^{6}} \int d \mathbf{q} d \mathbf{l} d \mathbf{m}, \\
& K_{\omega}^{0}(\mathbf{p}, \mathbf{l}, \mathbf{k}, \mathbf{m})|u(\mathbf{q})|^{2} K\left(\mathbf{l}+\mathbf{q}, \mathbf{p}^{\prime}, \mathbf{m}-\mathbf{q}, \mathbf{k}^{\prime}\right)
\end{aligned}
$$

Here $K_{\omega}^{0}\left(\mathbf{p}, \mathbf{p}^{\prime}, \mathbf{k}, \mathbf{k}^{\prime}\right)=\bar{G}_{\omega}\left(\mathbf{p}, \mathbf{p}^{\prime}\right) \bar{G}_{-\omega}\left(\mathbf{k}, \mathbf{k}^{\prime}\right)$ is the product of the two Green's functions $\bar{G}_{\omega}$ averaged over impurities separately. $K_{\omega}\left(\mathbf{p}, \mathbf{p}^{\prime}, \mathbf{k}, \mathbf{k}^{\prime}\right)=\overline{G_{\omega}\left(\mathbf{p}, \mathbf{p}^{\prime}\right) G_{-\omega}\left(\mathbf{k}, \mathbf{k}^{\prime}\right)}$ is the same product, where the Green's functions are averaged together. It corresponds to a term of the exact kernel of Eq. (5) related to the Matsubara frequency $\omega_{n}$. The exact kernel $K$ is the sum over the Matsubara frequencies $K=\sum_{\omega_{n}} K_{\omega_{n}}$.

Taking into account that the impurity scattering potential does not depend on the transmitted momentum q, Eq. (23) can be solved using the coordinate representation. The details of this solution are described in the Supplemental Material [34]. The kernel $K_{\omega_{n}}$ can be expressed as a matrix in the basis $\psi_{m}$ corresponding to the macroscopic coordinates $\mathbf{R}$ and in the basis $e^{i n \varphi}$ for the internal coordinate $\rho$. It is the same basis that we applied in the clean limit. Similar to the clean limit, the values $n$ and $m$ are related, $m=n+m_{0}$, where $m_{0}$ corresponds to the main order parameter.

$$
\begin{gathered}
K_{n, n^{\prime} ; \omega_{n}}=K_{n, n^{\prime} ; \omega_{n}}^{0}+\frac{1}{2 \pi \tau N(0)} K_{n, \emptyset ; \omega_{n}}^{0} K_{\emptyset, n^{\prime} ; \omega_{n}}^{0} \\
+\frac{1}{[2 \pi \tau N(0)]^{2}} K_{n, \emptyset ; \omega_{n}}^{0} K_{\emptyset, \emptyset ; \omega_{n}} K_{\emptyset, n^{\prime} ; \omega_{n}}^{0}, \\
K_{\emptyset, \emptyset ; \omega_{n}}=\frac{K_{\emptyset, \emptyset ; \omega_{n}}^{0}}{1-(2 \pi \tau N(0))^{-1} K_{\emptyset, \emptyset ; \omega_{n}}^{0}},
\end{gathered}
$$

where $\tau$ is the impurity scattering time and $N(0)=m^{*} / 2 \pi$ is $2 \mathrm{D}$ density of states. Here $\emptyset$ is the additional lower index corresponding to $n=0, \rho_{0}=0$. It appears due to the impurity scattering. The zero-order matrix elements $K_{n, n^{\prime} ; \omega_{n}}^{0}$ can be expressed as integrals,

$$
\begin{aligned}
K_{n, n^{\prime} ; \omega_{n}}^{0}= & \int \psi_{n+m_{0}}(\mathbf{R}) \psi_{n^{\prime}+m_{0}}\left(\mathbf{R}^{\prime}\right) e^{i n \varphi-i n^{\prime} \varphi^{\prime}} \\
& \times \bar{G}_{\omega_{n}}\left(\mathbf{R}+\boldsymbol{\rho} / 2, \mathbf{R}^{\prime}+\boldsymbol{\rho}^{\prime} / 2\right) \\
& \times \bar{G}_{-\omega_{n}}\left(\mathbf{R}-\boldsymbol{\rho} / 2, \mathbf{R}^{\prime}-\boldsymbol{\rho}^{\prime} / 2\right) \\
& \times \delta\left(|\boldsymbol{\rho}|-\rho_{0}\right) \delta\left(\left|\boldsymbol{\rho}^{\prime}\right|-\rho_{0}\right) d \boldsymbol{\rho} d \boldsymbol{\rho}^{\prime} d \mathbf{R} d \mathbf{R}^{\prime} .
\end{aligned}
$$

The value of $\rho_{0}$ should be considered to be $\rho_{0}=0$ when calculating $K_{n, n^{\prime} ; \omega_{n}}^{0}$ with the lower index $\emptyset$ with Eq. (26).

The integral equation for the order parameter is reduced to the matrix equation

$$
\Delta_{n}=T V_{0} \sum_{n^{\prime}, \omega_{n}} K_{n, n^{\prime} ; \omega_{n}} \Delta_{n^{\prime}}
$$

\section{The $\boldsymbol{n}=\mathbf{0}$ dominant channel}

When the dominant channel of pairing corresponds to $n=$ 0 , we apply $m_{0}=0$. It allows us to link indexes $m$ and $n$. After that, using Eqs. (24), (25), and (26), we can calculate matrix $K_{n, n^{\prime}}=\sum_{\omega} K_{n, n^{\prime} ; \omega}$ for $n, n^{\prime}=0,2$. The equation for $\Delta_{0}, \Delta_{2}$ has the form

$$
\left(\begin{array}{ll}
\mathcal{C}_{0,0} L_{00} & -\mathcal{C}_{0,2} L_{02} \\
-\mathcal{C}_{2,0} L_{20} & \mathcal{C}_{2,2} L_{22}
\end{array}\right)\left(\begin{array}{c}
\Delta_{0} \\
\Delta_{2}
\end{array}\right)=0 .
$$

Here the coefficients $\mathcal{C}_{n, n^{\prime}}$ are

$$
\begin{aligned}
\mathcal{C}_{0,0}= & \ln \frac{T}{T_{c 0}}-2 \sum_{n=0}^{\infty}\left[\frac{l l_{H} t I_{1}\left(y_{n}\right)}{l-l_{H} I_{1}\left(y_{n}\right)}-\frac{1}{2 n+1}\right], \\
\mathcal{C}_{2,0}= & \mathcal{C}_{0,2}=\sum_{n=0}^{\infty} \frac{l_{H} t}{2 \sqrt{2}} \frac{l I_{2}\left(y_{n}\right)}{l-l_{H} I_{1}\left(y_{n}\right)}, \\
\mathcal{C}_{2,2}= & \ln \frac{T}{T_{c 2}}-2 \sum_{n=0}^{\infty}\left[l_{H} t I_{3}\left(y_{n}\right)\right. \\
& \left.+\frac{l_{H}^{2} t}{8} \frac{I_{2}^{2}}{l-l_{H} I_{1}\left(y_{n}\right)}-\frac{1}{2 n+1}\right],
\end{aligned}
$$

and

$$
\begin{aligned}
& I_{1}(y)=\frac{1}{y} \int_{0}^{\infty} d x \exp \left[-x-(x / 2 y)^{2}\right] \\
& I_{2}(y)=\frac{1}{y^{3}} \int_{0}^{\infty} d x x^{2} \exp \left[-x-(x / 2 y)^{2}\right], \\
& I_{3}(y)=\frac{1}{y} \int_{0}^{\infty} d x \exp \left[-x-(x / 2 y)^{2}\right]\left(1-\frac{x^{2}}{y^{2}}+\frac{x^{4}}{8 y^{4}}\right) .
\end{aligned}
$$

Here $y_{n}=l_{H}[\operatorname{lt}(2 n+1)+1] / l, l$ is the mean free path measured in units of $L_{T_{c 0}}=v_{F} / 2 \pi T_{c 0}=0.882 \xi, l_{H}=L_{H} / L_{T_{c 0}}$, and $t=T / T_{c 0}$.

The upper critical field $H_{c 2}$ corresponds to the largest magnetic field when Eq. (28) has a nontrivial solution. Again, all the details about the pairing potential are dropped from the equation for $H_{c 2}$. The only information about the potential remains in critical temperatures $T_{c 0}$ and $T_{c 2}$.

The temperature dependence of the upper critical field $H_{c 2}(T)$ for different values of the mean free path $l$ and different values of $\ln \left(T_{c 0} / T_{c 2}\right)$ is presented in Fig. 4. The upper critical field is normalized to the extrapolation of the linear $H_{c 2}(T)$ dependence near $T_{c}: h_{c 2}=H_{c 2}(T) /\left(T_{c 0} d H_{c 2}\left(T_{c 0}\right) / d T\right)$. As can be seen from Fig. 4(a), there is a big difference in $H_{c 2}$ 

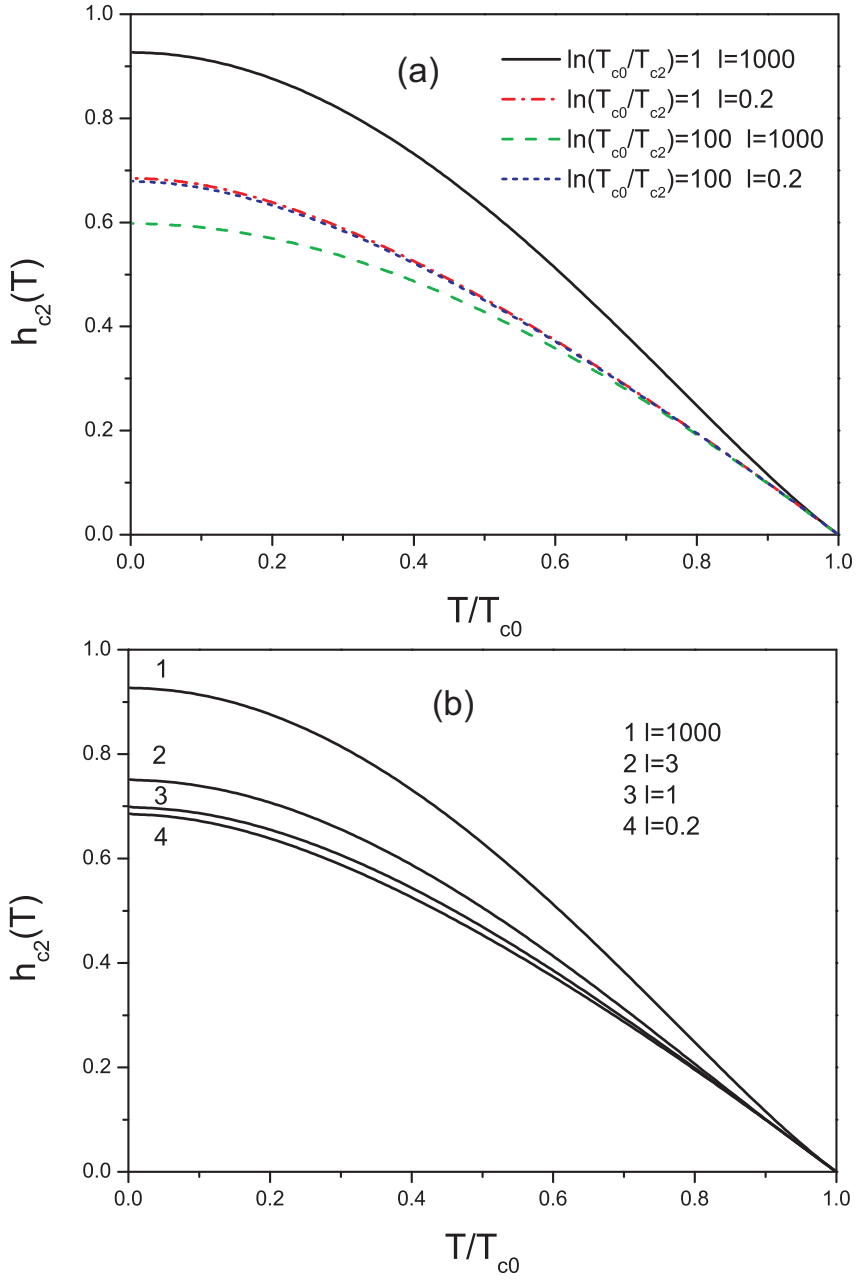

FIG. 4. (a) Upper critical field for different ratios of $T_{c 0} / T_{c 2}$ and different mean free paths of electrons $l$. (b) The dependence of the upper critical field on $l$ for $\ln \left(T_{c 0} / T_{c 2}\right)=1$.

between curves with $\ln \left(T_{c 0} / T_{c 2}\right)=1$ and $\ln \left(T_{c 0} / T_{c 2}\right)=100$ in the clean limit $l=1000$. On the other hand, these curves are almost indistinguishable in the dirty limit with $l=0.2$. Note that the curves in the dirty limit are below the orbital limiting field [Eq. (22)]. On the other hand, the curve of $H_{c 2}(T)$ in the clean limit and for $\ln \left(T_{c 0} / T_{c 2}\right)=1$ substantially exceeds the orbital limiting field. Therefore we can conclude that the finite radius of interaction substantially increases the critical field only in the clean limit. In the dirty limit the critical temperature in the channels with $n \neq 0$ is strongly suppressed by the impurity scattering, leading to suppression of the upper critical field. Figure 4(b) illustrates this suppression for finite values of $l$. The effect is significantly suppressed at $l \lesssim 3$.

\section{DISCUSSION}

The finite radius of the electron-electron interaction allows pairing with Cooper pairs with finite angular momentum $\Delta_{n}$. Each channel of pairing corresponds to its own critical temperature $T_{c n}$. However, even when the temperature is larger than $T_{c n}$ of some nondominant channel of the pairing, its order parameter can be generated in the presence of the external magnetic field. This generation is still related to the possibility

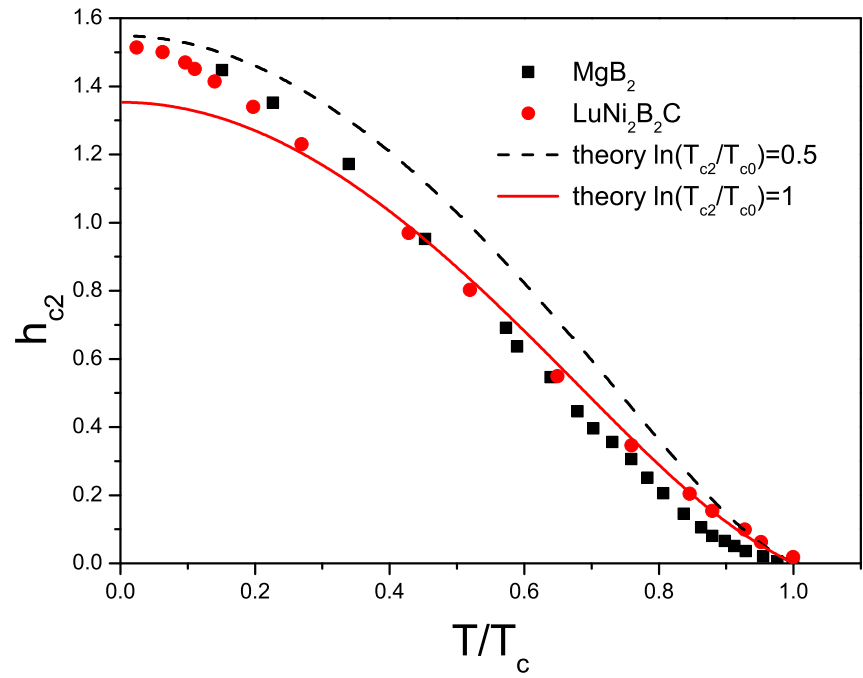

FIG. 5. Comparison of the theoretical model with the experiments on $\mathrm{MgB}_{2}$ [7] and on $\mathrm{LuNi}_{2} \mathrm{~B}_{2} \mathrm{C}$ [15].

of the existence of a given order parameter. For example, when the leading parameter corresponds to $n=0$ and $T_{c 2}$ tends to zero, $\ln \left(T_{c 0} / T_{c 2}\right) \rightarrow \infty$, and the effects of the coupling of $\Delta_{0}$ and $\Delta_{2}$ are absent (see Fig. 1).

The possibility of the existence of the order parameters with $n \neq 0$ is closely related to the conservation of the internal angular momentum of Cooper pairs. In the dirty limit the impurity scattering is much stronger than electron-electron attraction. The angular momentum of the Cooper pair is therefore quickly lost due to scattering. It suppresses not only the critical temperature of the nontrivial order parameters but also the generation of these parameters in the magnetic field.

A temperature dependence of $H_{c 2}$ similar to the one that results from our theory was observed in $\mathrm{MgB}_{2}$ [7] and in $\mathrm{LuNi}_{2} \mathrm{~B}_{2} \mathrm{C}$ [15]. In Fig. 5 we compare our theory with these experiments. The theory describes the experimental data relatively well. The small discrepancies can be attributed to the purely $2 \mathrm{D}$ character of the theory. We have discussed that the microscopic structure of the potential is reduced to the critical temperatures in different channels in terms of our theory. For most of the relevant values of $k_{F} \rho_{0}$ only two channels can have relatively high $T_{c n}$ and can effectively affect the $H_{c 2}(T)$ dependence with the selected coordinate form of the potential. Therefore the present theory can describe only the situations when the $H_{c 2}(T)$ dependence is governed by the interaction of two channels. However, more complex forms of the pairing potentials can allow three or more channels with relatively high $T_{c n}$. We believe that such potentials can lead to a further increase of the upper critical magnetic field at low temperatures.

In conclusion we generalized the Werthamer-HelfandHohenberg theory to the case of a nonlocal interaction between electrons. The theory is defined by the single parameter $k_{F} \rho_{0}$. We showed that when $k_{F} \rho_{0} \gtrsim 1$, the presence of the external magnetic field leads to the generation of additional components of the order parameter with different angular momenta. As a result the upper critical field is enhanced above the orbital limiting field. The upward curvature in the temperature dependence of $H_{c 2}(T)$ in the clean limit is predicted. The impurity scattering suppresses the effect in the dirty limit. 
[1] P. G. de Gennes, Superconductivity of Metals and Alloys (Addison-Wesley, New York, 1989).

[2] A. M. Clogston, Phys. Rev. Lett. 9, 266 (1962); B. S. Chandrasekhar, Appl. Phys. Lett. 1, 7 (1962).

[3] A. P. Mackenzie, S. R. Julian, G. G. Lonzarich, A. Carrington, S. D. Hughes, R. S. Liu, and D. C. Sinclair, Phys. Rev. Lett. 71, 1238 (1993).

[4] M. S. Osofsky, R. J. Soulen, Jr., S. A. Wolf, J. M. Broto, H. Rakoto, J. C. Ousset, G. Coffe, S. Askenazy, P. Pari, I. Bozovic, J. N. Eckstein, and G. F. Virshup, Phys. Rev. Lett. 71, 2315 (1993).

[5] Y. Ando, G. S. Boebinger, A. Passner, L. F. Schneemeyer, T. Kimura, M. Okuya, S. Watauchi, J. Shimoyama, K. Kishio, K. Tamasaku, N. Ichikawa, and S. Uchida, Phys. Rev. B 60, 12475 (1999).

[6] V. F. Gantmakher, G. E. Tsydynzhapov, L. P. Kozeeva, and A. N. Lavrov, J. Exp. Theor. Phys. 88, 148 (1999).

[7] L. Lyard, P. Samuely, P. Szabo, T. Klein, C. Marcenat, L. Paulius, K. H. P. Kim, C. U. Jung, H.-S. Lee, B. Kang, S. Choi, S.-I. Lee, J. Marcus, S. Blanchard, A. G. M. Jansen, U. Welp, G. Karapetrov, and W. K. Kwok, Phys. Rev. B 66, 180502(R) (2002); A. Gurevich, S. Patnaik, V. Braccini, K. H. Kim, C. Mielke, X. Song, L. D. Cooley, S. D. Bu, D. M. Kim, J. H. Choi, L. J. Belenky, J. Giencke, M. K. Lee, W. Tian, X. Q. Pan, A. Siri, E. E. Hellstrom, C. B. Eom, and D. C. Larbalestier, Supercond. Sci. Technol. 17, 278 (2004).

[8] C. Escribe-Filippini, J. Marcus, M. Affronte, H. Rakoto, J. M. Broto, J. C. Ousset, and S. Askenazy, Phys. C (Amsterdam, Neth.) 210, 133 (1993).

[9] E. Mun, N. Ni, J. M. Allred, R. J. Cava, O. Ayala, R. D. McDonald, N. Harrison, and V. S. Zapf, Phys. Rev. B 85, 100502(R) (2012); A. Pisoni, S. Katrych, P. Szirmai, B. Nafradi, R. Gaal, J. Karpinski, and L. Forro, J. Phys. Condens. Matter 28, 115701 (2016).

[10] T. Nakanishi, N. Motoyama, H. Mitamura, N. Takeshita, H. Takahashi, H. Eisaki, S. Uchida, and N. Mori, Int. J. Mod. Phys. B 14, 3617 (2000).

[11] I. J. Lee, P. M. Chaikin, and M. J. Naughton, Phys. Rev. B 62, R14669(R) (2000).

[12] D. D. Lawrie, J. P. Franck, J. R. Beamish, E. B. Molz, W.-m. Chert, and M. J. Graf, J. Low Temp. Phys. 107, 491 (1997).

[13] D. J. C. Walker, O. Laborde, A. P. Mackenzie, S. R. Julian, A. Carrington, J. W. Loram, and J. R. Cooper, Phys. Rev. B 51, 9375 (1995).
[14] A. S. Alexandrov, V. N. Zavaritsky, W. Y. Liang, and P. L. Nevsky, Phys. Rev. Lett. 76, 983 (1996).

[15] S. V. Shulga, S.-L. Drechsler, G. Fuchs, K.-H. Muller, K. Winzer, M. Heinecke, and K. Krug, Phys. Rev. Lett. 80, 1730 (1998).

[16] H. H. Wen, S. L. Li, and Z. X. Zhao, Phys. Rev. B 62, 716 (2000).

[17] N. Morozov, L. Krusin-Elbaum, T. Shibauchi, L. N. Bulaevskii, M. P. Maley, Yu. I. Latyshev, and T. Yamashita, Phys. Rev. Lett. 84, 1784 (2000).

[18] G. Grissonnanche, O. Cyr-Choinière, F. Laliberté et al., Nat. Commun. 5, 3280 (2014).

[19] A. Carrington, A. P. Mackenzie, and A. Tyler, Phys. Rev. B 54, R3788 (1996).

[20] M. Roulin, A. Junod, A. Erb, and E. Walker, J. Low Temp. Phys. 105, 1099 (1996).

[21] A. Junod, J.-Y. Genoud, G. Triscone, and T. Schneider, Phys. C (Amsterdam, Neth.) 294, 115 (1998).

[22] V. N. Zavaritsky, V. V. Kabanov, and A. S. Alexandrov, Europhys. Lett. 60, 127 (2002).

[23] R. Joynt, Phys. Rev. B 41, 4271 (1990).

[24] A. V. Balatsky, Phys. Rev. B 61, 6940 (2000).

[25] V. V. Kabanov, Phys. Rev. B 76, 172501 (2007); A. G. Lebed, Phys. Rev. Lett. 96, 037002 (2006).

[26] Y. Ren, J.-H. Xu, and C. S. Ting, Phys. Rev. B 53, 2249 (1996); W. Xu, W. Kim, Y. Ren, and C. S. Ting, ibid. 54, R12693 (1996).

[27] E. Helfand and N. R. Werthamer, Phys. Rev. Lett. 13, 686 (1964).

[28] E. Helfand and N. R. Werthamer, Phys. Rev. 147, 288 (1966).

[29] N. R. Werthamer, E. Helfand, and P. C. Hohenberg, Phys. Rev. 147, 295 (1966).

[30] A. A. Abrikosov, L. P. Gor'kov, and I. E. Dzyaloshinski, Methods of Quantum Field Theory in Statistical Physics (Dover Publications, Mineola, New York, USA, 1975).

[31] V. P. Mineev and K. V. Samokhin, Introduction to Unconventional Superconductivity (Gordon \& Breach Science Publishers, NY, 1999).

[32] J. B. Ketterson and S. N. Song, Superconductivity (Cambridge University Press, Cambridge, 1999).

[33] G. Rickayzen, Phys. Rev. 138, A73 (1965).

[34] See Supplemental Material at http://link.aps.org/supplemental/ 10.1103/PhysRevB.94.174506 for the solution of the Eq. (23) for the kernel with the presence of impurities. 